\title{
Bremsstrahlung vs. Thomson scattering in VUV-FEL plasma experiments
}

\author{
C. Fortmann 6 R. Redmer, H. Reinholz, G. Röpke, and A. Wierling \\ Inst. for Physics, Rostock University, 18051 Rostock, Germany \\ W. Rozmus \\ Dept. of Physics, University of Alberta, Edmonton, Canada
}

(Dated: October 29, 2018)

\begin{abstract}
We determine the spectral photon yield from a hot dense plasma irradiated by VUV-FEL light in a Thomson scattering experiment. The Thomson signal is compared to the emission background mainly caused by bremsstrahlung photons. We determine experimental conditions that allow for a signal-to-background ratio larger than unity. By derivation of the Thomson and the bremsstrahlung spectrum from linear response theory we present a consistent quantum statistical approach to both processes. This allows for a systematic treatment of medium and quantum effects such as dynamical screening and strong collisions. Results are presented for the threshold FEL-intensity as a function of density and temperature. We show that the account for quantum effects leads to larger thresholds as compared to previous work.
\end{abstract}

PACS numbers: 05.30.Fk, 52.25.Mq, 52.25.Os, 52.27.Aj, 52.70.-m, 71.45.Gm

Keywords: Thomson scattering, bremsstrahlung, free electron laser, plasma diagnostics, threshold intensity, dielectric function, dynamic structure factor

\section{INTRODUCTION}

Thomson scattering is a well established technique for experimental investigation of plasma parameters. Examples can be found in Refs. [1, 2, 3, 4, 5, 6]. Observables like particle density, temperature, composition, and degree of ionization can be spatially and temporally resolved by analysis of the scattering spectrum [7]. Until recently, coherent light sources have been available only for the visible and near UV part of the electromagnetic spectrum. Due to small critical density $n_{\text {crit }}=\omega^{2} \epsilon_{0} m_{\mathrm{e}} / e^{2} \approx 10^{20} \mathrm{~cm}^{-3}$ of free charge carriers for optical probes, the applicability of Thomson scattering using coherent sources has been limited to targets of relatively low density.

Glenzer et al. 8, 9] have shown and explored the possibility of x-ray Thomson scattering in solid density targets using the $\mathrm{Ti} \mathrm{He}-\alpha$ line at $4.75 \mathrm{keV}$ as probe light 10]. A new alternative emerged with the development of VUV-free electron lasers (VUV-FEL), providing pulses of coherent radiation in the far (vacuum-) ultraviolet. At the moment, the VUV-FEL at DESY Hamburg operates at $32 \mathrm{~nm}$ wavelength 11], corresponding to $38 \mathrm{eV}$ photons. With this coherent light source, dense matter up to solid densities of $10^{23} \mathrm{~cm}^{-3}$ can be penetrated, see Refs. 12, 13. . Under these conditions, the Thomson spectrum permits the determination of electron temperature and density directly from the position and height of collective resonances, i.e. plasmons, showing up in the scattering signal [9]. First experiments will be performed in the near future at the VUV-FEL facility at DESY at

*Electronic address: carsten.fortmann@uni-rostock.de URL: http://www.mpg.uni-rostock.de/vhvi104 $\lambda=32 \mathrm{~nm}$ FEL wavelength, while in future stages of the project, wavelengths from $13 \mathrm{~nm}$ (VUV-FEL) down to $0.1 \mathrm{~nm}$ (X-FEL) will be available.

Due to the large number of free charge carriers at the temperatures and densities considered, thermal bremsstrahlung emission, resulting from inelastic freefree scattering, contributes significantly to the emission background. Therefore, experimental conditions such as scattering angles, spectral properties of the probe and the detector have to be chosen as to obtain a maximum signal-to-background ratio. Background is to be understood as bremsstrahlung radiation, whereas signal corresponds to the photons having undergone Thomson scattering.

So far, classical formulas for the bremsstrahlung emission level going back to Kramers [14] have been used to determine threshold intensities of the external source to overcome the background due to bremsstrahlung [15, 16]. The Kramers result, given below in Eq. (19), is derived from the assumption of Keplerian trajectories of the emitting electron in the Coulomb field of an ion and integration over all initial velocities weighted with the Maxwellian velocity distribution function. Quantum features are only accounted for in a semiclassical way: The velocity integral extends over velocities $v$, fulfilling the condition $m v^{2} / 2 \geq \hbar \omega$, i.e. the kinetic energy has to be larger than the photon energy. Further quantum properties, such as the finite photon momentum as well as the quantum mechanical nature of the scattering process are not accounted for. By comparing the Thomson signal strength at the laser wavelength $\lambda=14.7 \mathrm{~nm}$ to the bremsstrahlung photon yield calculated from Kramers formula, Baldis et al. find threshold intensities of $10^{13} \mathrm{~W} / \mathrm{cm}^{2}$ for typical values of free electron density $n_{\mathrm{e}}=10^{22} \mathrm{~cm}^{-3}$ and temperature $k_{\mathrm{B}} T=100 \mathrm{eV}[16$. 
In this work, we evaluate threshold conditions (intensities) using improved expressions for the bremsstrahlung spectrum. As usual, corrections to Kramers formula for bremsstrahlung are described by the so-called Gaunt factor [17]. In the simplest approach it is obtained by taking into account collisions between electrons and fixed ions in Born approximation. In dense plasmas, many-particle effects as dynamical screening become important. Moreover, strong collisions have to be accounted for. We show in this paper how the Gaunt factor can be derived from linear response theory [18] in a general way. Within this framework, modifications of the emission spectrum beyond Born aproximation can be included in a systematic manner [19] as will be discussed later.

We then apply our formulas to determine the threshold intensities for a broad range of experimental parameters (wavelength, spectral properties of detectors, and different materials), relevant for future experiments at DESY. Furthermore we compare Thomson and bremsstrahlung photon yield over a finite spectral range. Thereby, and by taking improved expressions for the bremsstrahlung cross section, we show, that even higher thresholds have to be reached in order to obtain a Thomson signal above the bremsstrahlung level at least near the plasmon resonances. These peaks are much lower than the central peak being essentially an ion feature.

The present work is organized as follows: In the first section we review the basic physics of Thomson scattering and bremsstrahlung and how they can be expressed in terms of the dynamic structure factor and the dielectric function, respectively. Since these two quantities are related to each other via the fluctuation-dissipation theorem [20], we are able to describe both processes on a common and consistent basis.

We then compare the emission level due to bremsstrahlung to the Thomson signal whose strength is proportional to the flux of incoming photons, i.e. the power density of the external source. Thereby, we find expressions for the threshold power density as a function of particle density and temperature. In the last section we discuss our results for various sets of experimental parameters relevant for future experiments at the VUVFEL.

\section{THOMSON SCATTERING AND BREMSSTRAHLUNG}

The central quantity of interest is the spectral power density $\mathrm{d} P / \mathrm{d} V \mathrm{~d} \lambda \mathrm{d} \Omega$, i.e. the rate of energy radiated per unit scattering volume $\mathrm{d} V$, wavelength $\mathrm{d} \lambda$, and solid angle $d \Omega$. The total spectral power density is the sum of the corresponding quantity for every radiative process in the plasma. In this work we focus on Thomson scattering and bremsstrahlung, i.e.

$$
\frac{\mathrm{d}^{3} P_{\mathrm{tot}}}{\mathrm{d} V \mathrm{~d} \lambda \mathrm{d} \Omega}=\frac{\mathrm{d}^{3} P_{\mathrm{Th}}}{\mathrm{d} V \mathrm{~d} \lambda \mathrm{d} \Omega}+\frac{\mathrm{d}^{3} P_{\mathrm{br}}}{\mathrm{d} V \mathrm{~d} \lambda \mathrm{d} \Omega} .
$$

To unambiguously identify the Thomson signal, we require that the Thomson power density is at least equal to the bremsstrahlung level,

$$
\frac{\mathrm{d}^{3} P_{\mathrm{Th}}}{\mathrm{d} V \mathrm{~d} \lambda \mathrm{d} \Omega} \geq \frac{\mathrm{d}^{3} P_{\mathrm{br}}}{\mathrm{d} V \mathrm{~d} \lambda \mathrm{d} \Omega} .
$$

The Thomson spectrum is given by the intensity of the probe laser $I_{\mathrm{L}}$, and the Thomson scattering cross section $\mathrm{d}^{2} \sigma_{\mathrm{Th}} / \mathrm{d} \omega \mathrm{d} \Omega$. To account for the finite spetral bandwidth of the detector, one has to convolute each power spectrum with a detector function $G(\lambda)$. In practice, this is only relevant for the Thomson signal since the bremsstrahlung spectrum is slowly varying in the relevant frequency region. The Thomson power spectrum reads

$$
\frac{\mathrm{d}^{3} P_{\mathrm{Th}}(\lambda)}{\mathrm{d} V \mathrm{~d} \lambda \mathrm{d} \Omega}=I_{\mathrm{L}} \int \mathrm{d} \bar{\lambda} G(\lambda-\bar{\lambda}) \frac{\mathrm{d}^{2} \sigma_{\mathrm{Th}}\left(\omega_{\bar{\lambda}}\right)}{\mathrm{d} \Omega \mathrm{d} \omega}=I_{\mathrm{L}} \bar{R}(\lambda) .
$$

We have introduced the response function $\bar{R}(\lambda)$, where the bar denotes the convolution with the detector function. Note that we assume an optically thin plasma, thus radiation transport is neglected. Also, due to the short pulselength of the VUV-FEL (20-120 fs), we neglect the heating of the plasma due to the probe beam.

The bremsstrahlung spectrum does solely depend on the plasma parameters density and temperature, so that, with a suitable formula for the bremsstrahlung spectrum, which we will abbreviate by the notation $\mathrm{d}^{3} P_{\mathrm{br}} / \mathrm{d} V \mathrm{~d} \lambda \mathrm{d} \Omega \equiv j(\lambda)$ in the following, Eq. (2) defines a threshold intensity

$$
I_{\text {thresh }}(\lambda)=\frac{j(\lambda)}{\bar{R}(\lambda)} .
$$

We will now briefly describe how expressions for the Thomson scattering and the bremsstrahlung spectrum can be obtained from a common starting point, i.e. the dielectric function of the plasma.

\section{A. Thomson scattering}

The cross section for Thomson scattering in a plasma can be given in terms of the dynamic structure factor (DSF), $S(\boldsymbol{k}, \omega)$ :

$$
\frac{\mathrm{d}^{2} \sigma_{\mathrm{Th}}(\boldsymbol{k}, \omega)}{\mathrm{d} \Omega \mathrm{d} \omega}=\left(\frac{\mathrm{d} \sigma(\Omega)}{\mathrm{d} \Omega}\right)_{\mathrm{Th}} \frac{k_{1}}{k_{0}} S(\boldsymbol{k}, \omega),
$$

see Ref. 21, 22] for details. The variables $(\boldsymbol{k}, \omega)$ are related to the transferred linear momentum and energy, respectively, while $k_{0}=\omega_{0} / c$ and $k_{1}=\left|\boldsymbol{k}_{\mathbf{0}}+\boldsymbol{k}\right|$ are incoming and outgoing linear momenta of the laser field, respectively. $(\mathrm{d} \sigma(\Omega) / \mathrm{d} \Omega)_{\mathrm{Th}}$ is the Thomson scattering cross section for the isolated scattering event. It is given by the Klein-Nishina formula, its derivation can be found in standard textbooks of quantum electrodynamics, e.g. Ref. [23]. 
In the nonrelativistic limit, the Thomson cross section for unpolarized light is given by

$$
\left(\frac{\mathrm{d} \sigma}{\mathrm{d} \Omega}\right)_{\mathrm{Th}}=\frac{r_{\mathrm{e}}^{2}}{2}\left(1+\cos ^{2} \theta\right) .
$$

Here, $r_{\mathrm{e}}=e^{2} / 4 \pi \epsilon_{0} m_{\mathrm{e}} c^{2}$ is the classical electron radius, $\theta$ is the scattering angle between by $\boldsymbol{k}_{1}$ and $\boldsymbol{k}_{0}$.

Equation (5) shows that plasma collective properties can be measured in a Thomson scattering experiment. However, this requires an accurate theory of the dynamic structure factor. The dynamic structure factor $S(\boldsymbol{k}, \omega)$ is closely related to the longitudinal dielectric function by the fluctuation-dissipation theorem [24]

$$
S(\boldsymbol{k}, \omega)=-\frac{\epsilon_{0} \hbar k^{2}}{\pi e^{2} n_{\mathrm{e}}} \frac{\operatorname{Im} \epsilon_{l}^{-1}(\boldsymbol{k}, \omega)}{1-\exp \left(-\hbar \omega / k_{\mathrm{B}} T\right)} .
$$

This relation can be utilized by applying an appropriate approximation to the dielectric function. Due to the mass ratio $m_{\mathrm{e}} / m_{\mathrm{i}} \ll 1$, Thomson scattering on ions can be neglected. Therefore, we will approximate $S(\boldsymbol{k}, \omega)$ by $S_{\text {ee }}(\boldsymbol{k}, \omega)$, the electronic DSF.

\section{B. Bremsstrahlung}

Radiative free-free transitions of electrons, known as bremsstrahlung, represent the main source of emission in a hot, fully ionized plasma. However, the bremsstrahlung process requires the presence of a scattering partner that carries the recoil momentum. Kinematically, emission by electrons which scatter on ions is dominant.

For a thermally equilibrated plasma, emission, characterized by the spectral power density $j(\omega)$, and the absorption coefficient $\alpha(\omega)$ as the relative attenuation of the intensity of electromagnetic waves propagating in the medium per unit length, are linked by Kirchhoff's law 25] $j(\omega)=L(\omega) \alpha(\omega)$, with the Planck distribution $L(\omega)=\hbar \omega^{3} /\left[4 \pi^{3} c^{2}\left(\exp \left(\hbar \omega / k_{\mathrm{B}} T\right)-1\right)\right]$. Note that we consider the long wavelength limit, thus the wavevector $\boldsymbol{k}$ does not show up as an argument of the quantities $j(\omega)$ and $\alpha(\omega)$. The absorption coefficient can be obtained from the imaginary part of the transverse dielectric function according to

$$
\alpha(\omega)=\frac{\omega}{c} \frac{\operatorname{Im} \epsilon_{t}(\omega)}{n(\omega)},
$$

where the index of refraction $n(\omega)$ is also linked to the transverse dielectric function by

$$
n(\omega)=\frac{1}{\sqrt{2}}\left(\operatorname{Re} \epsilon_{t}(\omega)+\left|\epsilon_{t}(\omega)\right|\right)^{1 / 2} .
$$

In the long wavelength limit, the longitudinal dielectric function, which appears in the fluctuation-dissipation theorem (77), and the transverse dielectric function coincide [26]. Thus, relations (7) and (8) enable us to treat both Thomson scattering and bremsstrahlung on a common basis, namely by using an appropriate theory for the longitudinal dielectric function.

\section{Consistent approximations}

The aim of this work is to compare both radiation processes, Thomson scattering and bremsstrahlung, calculated in a consistent approximation. The comparison has to be carried out between the contributions of either process in leading order of density.

Bremsstrahlung occurs in second order of the coupling constant $\alpha_{\mathrm{QED}}=1 / 137$ as can be seen from the transition amplitude $w_{\mathrm{fi}}^{\text {br }}$ expressed by Feynman diagrams,

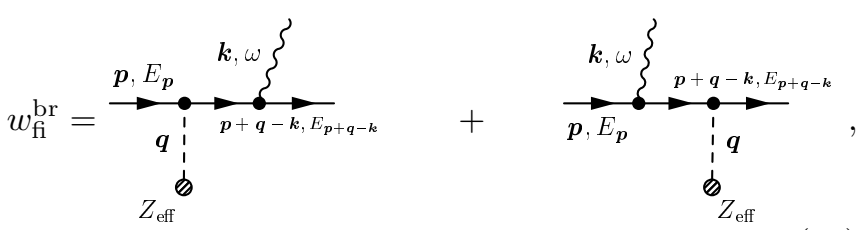

giving the Bethe-Heitler cross section, see Ref. 23]. The transition amplitude involves a longitudinal field (Coulomb field) i.e. a scattering partner, say an ion of effective charge $Z_{\text {eff }}$. Thus, bremsstrahlung is naturally of second order in density. A free electron does not emit bremsstrahlung, unless collisions take place. The challenge is then to accuratly describe the scattering process itself.

Born approximation, as given by Eq. (10) does not describe the correct behaviour of the bremsstrahlung spectrum in the case of collisions involving high transfer momenta, so-called strong scatterings. These can be included by ladder-summation of all one-photon exchange processes, which leads to the t-matrix. Thereby, the electron-ion interaction is treated accuratly in all orders. In the Coulomb limit, one finds Sommerfeld's expression for bremsstrahlung, see Eq. (22) below, and Ref. [27]. On the other hand, due to the long range behaviour of the Coulomb potential, Born approximation as well as the Sommerfeld result suffer from infrared divergencies. Here, the account for screening leads to convergent results. For details, we refer to Ref. 19]. In our calculations we will use Sommerfeld's formula Eq. (22). Since we are interested in the bremsstrahlung spectrum at high frequencies, i.e. in the vicinity of the FEL frequency $\left(\hbar \omega_{0}=38 \mathrm{eV}\right)$, no screening effects are considered here. Note that the use of the t-matrix does not increase the order in density, instead, it gives the accurate Gaunt factor in leading order of density. Further many-particle effects like self-energy and vertex corrections lead to higher order contributions in density and, therefore, are not considered here. For a consistent treatment of the self-energy of the scattering electron, given by multiple scattering on ions and its impact on the bremsstrahlung spectrum, see Ref. [28].

In the case of Thomson scattering, we adopt the same strategy: We calculate the contribution from lowest order in density. Unlike bremsstrahlung, already the first order in density gives a finite contribution. The amplitude for 
Thomson scattering in second order of the interaction

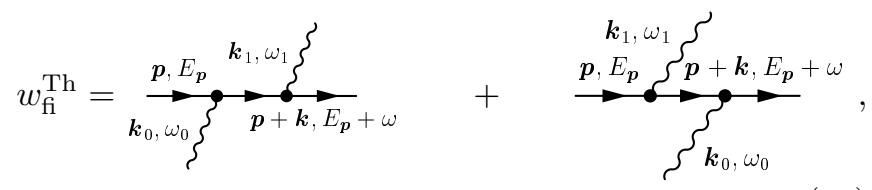

does not contain any longitudinal field and therefore behaves regularly over the whole spectral range.

Thus, the DSF, which describes the Thomson scattering in the medium, is taken in random phase approximation (RPA) that accounts for the dynamical screening. This is all the more important, as the Thomson scattering cross section is evaluated in the vicinity of the plasma frequency where dynamical screening is the dominant effect [20]. Collisions are neglected, since they occur in the next to leading order in density.

To conclude, a consistent comparison between Thomson scattering and bremsstrahlung in media asks for the cross section of either process in leading order in density. This consideration leads us to the RPA for the dynamic structure factor, and a t-matrix ladder summation for the bremsstrahlung spectrum.

\section{LINEAR RESPONSE THEORY}

Thomson scattering and bremsstrahlung can be determined by the longitudinal dielectric function $\epsilon_{l}(\boldsymbol{k}, \omega)$. We will now briefly outline how the longitudinal dielectric function can be obtained in the framework of linear response theory. A discussion of its fundamental aspects can be found in [18], for various applications to optical properties of plasmas, see [20]. In general, the longitudinal dielectric function $\epsilon_{l}(\boldsymbol{k}, \omega)$ is given in terms of the dielectric susceptibility $\chi_{c c^{\prime}}(\boldsymbol{k}, \omega)$, where $c$ and $c^{\prime}$ label the different species in the plasma and further quantum numbers such as spin,

$$
\epsilon_{l}(\boldsymbol{k}, \omega)=\frac{1}{1-\sum_{c c^{\prime}} V_{c c^{\prime}}(\boldsymbol{k}) \chi_{c c^{\prime}}(\boldsymbol{k}, \omega)} .
$$

$V_{c c^{\prime}}(\boldsymbol{k})$ is the unscreened Coulomb potential between particles of species $c$ and $c^{\prime}$,

$$
V_{c c^{\prime}}(\boldsymbol{k})=\frac{q_{c} q_{c^{\prime}}}{\epsilon_{0} k^{2}}
$$

Within linear response theory, the Kubo formula relates the susceptibility (response function) to the currentcurrent correlation function 26]

$$
\chi_{c c^{\prime}}(\boldsymbol{k}, \omega)=i \beta \Omega_{0} \frac{k^{2}}{\omega q_{c} q_{c^{\prime}}}\left\langle J_{k, c}^{z} ; J_{k, c^{\prime}}^{z}\right\rangle_{\omega+i \eta},
$$

where $\beta=1 / k_{\mathrm{B}} T$ is the inverse temperature, $\Omega_{0}$ is a normalization volume. The current operator for particles of species $c$ is defined as

$$
\boldsymbol{J}_{k, c}=\frac{1}{\Omega_{0}} \sum_{\boldsymbol{p}} \frac{q_{c}}{m_{c}} \hbar \boldsymbol{p} a_{\boldsymbol{p}-\boldsymbol{k} / 2, c}^{\dagger} a_{\boldsymbol{p}+\boldsymbol{k} / 2, c} .
$$

One expresses the current-current correlation function by a force-force correlation function $\left\langle\dot{J}_{k, c}^{z} ; \dot{J}_{k, c^{\prime}}^{z}\right\rangle_{\omega+i \eta}$, using the time derivative of the current $\dot{\boldsymbol{J}}_{k, c}=i\left[H, \boldsymbol{J}_{k, c}\right] / \hbar$. The force-force correlation function is more suited for a perturbative treatment, see Ref. 20] and App. A] where also definitions and useful properties of correlation functions are given.

\section{CALCULATION OF THE BREMSSTRAHLUNG SPECTRUM}

Since the bremsstrahlung spectrum is evaluated at frequencies far above the plasma frequency, we can use the high frequency limit of Eq. (12) in order to derive the absorption coefficient. For details, we refer to App. A In the high frequency limit, the absorption coefficient (8) is given by [19, 20]

$\alpha(\omega)=\frac{\beta \Omega_{0}}{c \epsilon_{0} \omega^{2}} \operatorname{Re}\left\langle\dot{j}_{0, \mathrm{e}}^{z}, \dot{J}_{0, \mathrm{e}}^{z}\right\rangle_{\omega+i \eta}=\frac{\pi \Omega_{0}}{c \epsilon_{0} \omega^{3}} \operatorname{Im} G_{j j}(\omega+i \eta)$.

Here we introduced the force-force Green function $G_{j j}(\omega)$, defined in Eq. A6 , which can conveniently be calculated using Feynman diagrams, see Fig. 11 (a). $G_{4}$

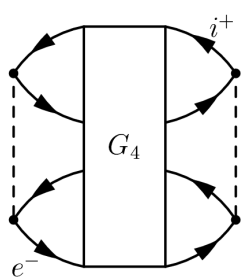

(a)

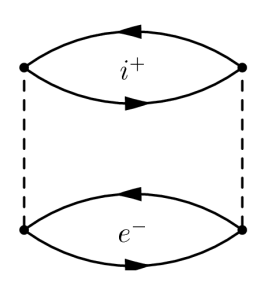

(b)

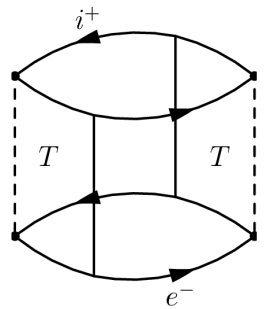

(c)
FIG. 1: Diagrammatic representation of $G_{j j}\left(\omega_{\mu}\right)$. (a) full account of all medium effects by a four particle Green function, (b) Born approximation, (c) t-matrix approximation.

denotes a four-particle Green function that contains all interactions between electrons and ions [19]. In the long wavelength limit considered here, e-e and i-i collisions vanish due to momentum conservation. We perform a sequence of approximations by selecting certain diagrams contributing to $G_{4}$.

\section{A. Born approximation}

As the lowest order contribution with respect to the interaction potential, Fig. 1(b) shows the Born approximation. This approach reproduces the well-known Bethe- 
Heitler formula in the nonrelativistic limit:

$$
\begin{aligned}
& j^{\mathrm{B}}(\omega)= \frac{16 Z_{\mathrm{eff}}^{2} n_{\mathrm{i}} n_{\mathrm{e}}}{3 c^{3}}\left(\frac{e^{2}}{4 \pi \epsilon_{0}}\right)^{3}\left(\frac{\beta}{2 \pi m_{\mathrm{e}}}\right)^{3 / 2} \times \\
& \quad \times \int_{\hbar \omega}^{\infty} \mathrm{d} E_{p}^{\mathrm{e}} \mathrm{e}^{-\beta E_{p}^{\mathrm{e}}} \ln \left|\frac{\sqrt{E_{p}^{\mathrm{e}}}+\sqrt{E_{p}^{\mathrm{e}}-\hbar \omega}}{\sqrt{E_{p}^{\mathrm{e}}}-\sqrt{E_{p}^{\mathrm{e}}-\hbar \omega}}\right| \\
&=\frac{8 Z_{\mathrm{eff}}^{2} n_{\mathrm{i}} n_{\mathrm{e}}}{3 m_{\mathrm{e}} c^{3} \pi}\left(\frac{e^{2}}{4 \pi \epsilon_{0}}\right)^{3} \sqrt{\frac{\beta}{2 \pi m_{\mathrm{e}}}} \mathrm{e}^{-\beta \hbar \omega / 2} K_{0}(\beta \hbar \omega / 2) .
\end{aligned}
$$

$E_{\boldsymbol{p}}^{c}$ is the free particle energy, for nonrelativistic particles $E_{\boldsymbol{p}}^{c}=\hbar^{2} p^{2} / 2 m_{c}$ holds. Details of the calculation are discussed in [28]. In Eq. (17) the effective ion charge $Z_{\text {eff }}$ is used to account for the screening of the charge $Z e$ of the nucleus due to inner shell electrons. In Ref. [16], $Z_{\text {eff }}$ is calculated as a function of temperature and density in the framework of Thomas-Fermi theory, see Ref. [29] for details. Alternatively, $Z_{\text {eff }}$ can be obtained in the socalled chemical picture by solving Saha's equation, see Ref. 30]. Especially for high temperatures, the chemical picture gives more reliable results, as the ionization equilibria between different ionization stages are satisfied. We will therefore use the chemical picture in this work and calculate the mean ionization level with COMPTRA04 [30]. The Thomas-Fermi model will only be applied for the purpose of comparison to results obtained by Baldis et al. in Ref. 16].

The logarithm in Eq. (17) is the nonrelativistic limit of the Bethe-Heitler cross section [31, 32]. The drawback of this result is the divergence of the bremsstrahlung spectrum in the limit $\omega \rightarrow 0$. However, physically this is of no importance, since for low frequencies the index of refraction is different from unity and modifies the spectrum significantly. This is known as dielectric suppression [33].

It is common use to write formulas for the emission and absorption due to (inverse) bremsstrahlung in terms of Kramers classical result [14]

$$
j^{\mathrm{K}}(\omega)=\frac{8 Z_{\mathrm{eff}}^{2} n_{\mathrm{e}} n_{\mathrm{i}}}{3 m_{\mathrm{e}} c^{3}}\left(\frac{e^{2}}{4 \pi \epsilon_{0}}\right)^{3}\left(\frac{\beta}{6 \pi m_{\mathrm{e}}}\right)^{1 / 2} \exp (-\beta \hbar \omega),
$$

multiplied with a correction factor $\bar{g}_{\mathrm{ff}}(\omega)$, called Gauntfactor [17], which takes into account medium effects as well as quantum corrections, i.e.

$$
j(\omega)=j^{\mathrm{K}}(\omega) \cdot \bar{g}_{\mathrm{ff}}(\omega)
$$

With Eq. (18), the Gaunt factor in Born approximation reads

$$
\bar{g}_{\mathrm{ff}}^{\mathrm{B}}(\omega)=\frac{\sqrt{3}}{\pi} \exp (\beta \hbar \omega / 2) K_{0}(\beta \hbar \omega / 2)
$$

\section{B. Strong collisions}

The Born approximation assumes free particles as the in- and out states in the scattering amplitude. Taking the true scattering states, i.e Coulomb wavefunctions, leads to the so-called Sommerfeld formula [27],

$$
\begin{aligned}
\bar{g}_{\mathrm{ff}}^{\mathrm{S}}(\omega, T)= & \frac{1}{k_{\mathrm{B}} T} \int_{0}^{\infty} \mathrm{d} E_{i} \mathrm{e}^{-E_{i} / k_{\mathrm{B}} T} g_{\mathrm{ff}}^{\mathrm{S}}\left(\omega, E_{i}\right), \\
\text { with } & \begin{aligned}
g_{\mathrm{ff}}^{\mathrm{S}}\left(\omega, E_{i}\right)= & \frac{4 \sqrt{3}}{\pi}\left[\frac{\left(\eta_{i}^{2}+\eta_{f}^{2}+2 \eta_{i}^{2} \eta_{f}^{2}\right)}{2 \eta_{i} \eta_{f}} I_{0}-\left(1+\eta_{i}^{2}\right)^{1 / 2}\left(1+\eta_{f}^{2}\right)^{1 / 2} I_{1}\right] I_{0}, \\
\text { and } & \\
I_{l}= & \frac{1}{4}\left[\frac{4 \sqrt{E_{i} E_{f}}}{\left(\sqrt{E_{i}}-\sqrt{E_{f}}\right)^{2}}\right]^{l+1}\left|\frac{\sqrt{E_{i}}-\sqrt{E_{f}}}{\sqrt{E_{i}}+\sqrt{E_{f}}}\right|^{i\left(\eta_{i}+\eta_{f}\right)} \mathrm{e}^{\pi\left|\eta_{i}-\eta_{f}\right| / 2} \frac{\left|\Gamma\left(l+1+i \eta_{i}\right) \Gamma\left(l+1+i \eta_{f}\right)\right|}{\Gamma(2 l+2)} \times \\
& \times{ }_{2} F_{1}\left(l+1-i \eta_{f}, l+1-i \eta_{i} ; 2 l+2 ;-\frac{4 \sqrt{E_{i} E_{f}}}{\left(\sqrt{E_{i}}-\sqrt{E_{f}}\right)^{2}}\right), \quad E_{f}=E_{i}-\hbar \omega .
\end{aligned}
\end{aligned}
$$

$\eta_{i / f}^{2}=Z_{\text {eff }}^{2} \mathrm{Ry} / E_{i / f}$ is the Sommerfeld parameter with the Rydberg energy Ry $=m_{\mathrm{e}} e^{4} / 2\left(4 \pi \epsilon_{0} \hbar\right)^{2} \simeq 13.6 \mathrm{eV}$, ${ }_{2} F_{1}(a, b ; c ; d)$ is the hypergeometric function 34 and
$\Gamma(x)$ is the Gamma function. As shown in Ref. [19], Sommerfeld's expression is also obtained by a t-matrix ladder summation with a statically screened Debye potential (Fig. 1(c)) if the limit of vanishing inverse screen- 
ing length (Coulomb limit) is taken. It gives the correct Gaunt factor in the low density limit, which is considered here.

Fig. 2 shows the dependence of the Gaunt factor on the photon energy for electron temperatures $k_{\mathrm{B}} T=$ $10 \mathrm{eV}, 100 \mathrm{eV}$ and $1000 \mathrm{eV}$. Over the large energy interval shown, the Gaunt factor in either calculation for a fixed temperature does not vary much and is of order unity, thus the widely used approximation to set $\bar{g}_{\mathrm{ff}}(\omega) \approx 1$ is justified for estimates of the emission level as done in Refs. 15, 16]. More specific, $\bar{g}_{\mathrm{ff}}(\omega)=1$ is a good approximation for photon energies comparable to the temperature $\hbar \omega / k_{\mathrm{B}} T \simeq 1$ and for temperatures in the vicinity of the ionization energy $\left(k_{\mathrm{B}} T \simeq Z^{2} \mathrm{Ry}\right.$ for hydrogenic systems). If one of these conditions is not met, one should use the full Sommerfeld expression (22) or appropriate approximations, see the detailed discussion in Ref. [35]. For the VUV-FEL experiments, both requirements are satisfied only roughly: The laser provides photon energies of $\hbar \omega \simeq 40 \mathrm{eV}$ and the optical pump laser will excite the plasma to temperatures of $k_{\mathrm{B}} T \simeq 10 \ldots 50 \mathrm{eV}$, which is of almost the same order of magnitude as the first ionization energies of aluminum $(6 \mathrm{eV}, 19 \mathrm{eV}, 28 \mathrm{eV})$, or hydrogen $(13.6 \mathrm{eV})$ [36], materials presumably used as target in the experiments. Thus we take into account the Gaunt factor in t-matrix approximation (Eq. (22)) in our calculations.

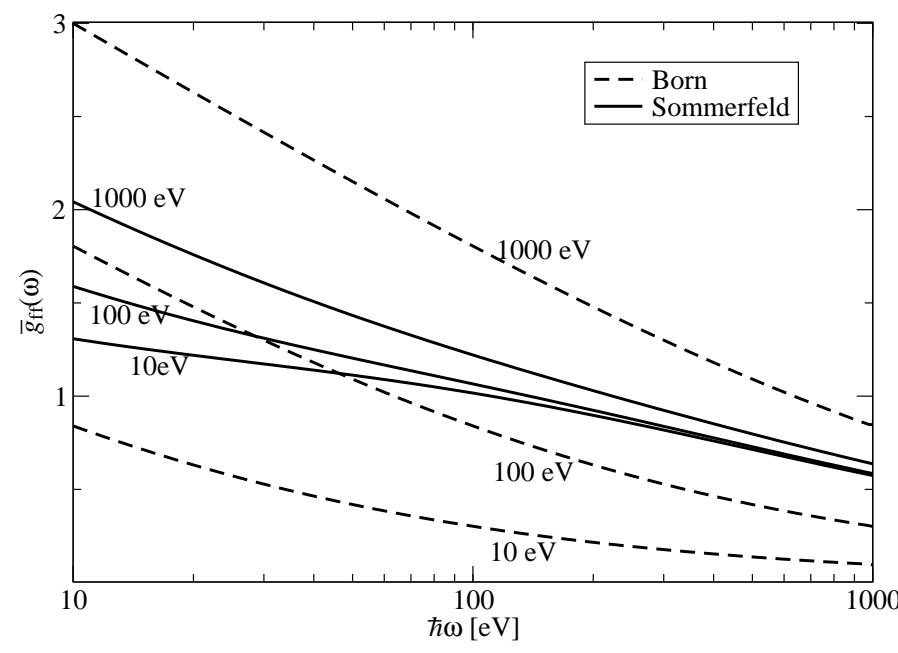

FIG. 2: Gaunt factor $\bar{g}_{\text {ff }}(\omega)$ in Born approximation Eq. 21] and using Sommerfeld's formula Eq. (22), respectively. Results are presented as a function of the photon energy $\hbar \omega$ for various temperatures.

In Ref. [19], a consistent treatment of the impact of dynamical screening and strong collisions on bremsstrahlung based on the Gould-DeWitt scheme [37] is given. It is shown that the high frequency behaviour of the Gaunt factor is dominated by the t-matrix contribution, whereas dynamical screening can be neglected as long as the considered frequencies are large compared to the plasma frequency.

\section{DYNAMIC STRUCTURE FACTOR}

The total dynamic structure factor is defined as 38

$$
S_{c c}(\boldsymbol{k}, \omega)=\frac{1}{2 \pi N} \int_{-\infty}^{+\infty} \mathrm{d} t \mathrm{e}^{i \omega t}\left\langle\rho_{c}(\boldsymbol{k}, t) \rho_{c}(-\boldsymbol{k}, 0)\right\rangle
$$

$\langle\ldots\rangle$ denotes the ensemble equilibrium average. Here, our discussion is focused on the electron structure factor $S_{\text {ee }}(\boldsymbol{k}, \omega)$. The fluctuation-dissipation theorem (77) in connection with Eq. (12) allows to express the electronic part of the dynamical structure factor via the electronic dielectric susceptibility $\chi_{\mathrm{ee}}(\boldsymbol{k}, \omega)$,

$$
S_{\mathrm{ee}}(\boldsymbol{k}, \omega)=\frac{\hbar}{\pi n_{\mathrm{e}}} \frac{\operatorname{Im} \chi_{\mathrm{ee}}(\boldsymbol{k}, \omega)}{1-\exp \left(-\hbar \omega / k_{\mathrm{B}} T_{\mathrm{e}}\right)} .
$$

We will consider the classical limit, where the denominator of Eq. (26) can be approximated by $\hbar \omega / k_{\mathrm{B}} T_{\mathrm{e}}$. In RPA, the following equation for the susceptibility holds [39]:

$\chi_{c c^{\prime}}^{\mathrm{RPA}}(\boldsymbol{k}, \omega)=\chi_{c}^{0}(\boldsymbol{k}, \omega) \delta_{c c^{\prime}}+\chi_{c}^{0}(\boldsymbol{k}, \omega) V_{c c^{\prime}}^{\mathrm{sc}}(\boldsymbol{k}, \omega) \chi_{c^{\prime}}^{0}(\boldsymbol{k}, \omega)$.

$V^{\mathrm{sc}}(\boldsymbol{k}, \omega)$ is the screened interaction potential which acts between particles of species $c$ and $c^{\prime}$. It satisfies the equation

$$
V_{c c^{\prime}}^{\mathrm{sc}}(\boldsymbol{k}, \omega)=V_{c c^{\prime}}(\boldsymbol{k})+\sum_{d} V_{c d}(\boldsymbol{k}) \chi_{d}^{0}(\boldsymbol{k}, \omega) V_{d c^{\prime}}^{\mathrm{sc}}(\boldsymbol{k}, \omega) .
$$

The free susceptibility $\chi_{c}^{0}(\boldsymbol{k}, \omega)$ is obtained from Eq. (14), taking the current-current correlation function only in zeroth order with respect to the interaction, i.e.

$$
\begin{aligned}
\chi_{c}^{0}(\boldsymbol{k}, \omega) & =i \beta \Omega_{0} \frac{k^{2}}{\omega q_{c} q_{c^{\prime}}}\left\langle J_{k, c}^{z} ; J_{k, c^{\prime}}^{z}\right\rangle_{\omega+i \eta}^{0} \\
= & \delta_{c c^{\prime}} \sum_{p} \frac{f_{\boldsymbol{p}+\boldsymbol{k} / 2}^{c}-f_{\boldsymbol{p}-\boldsymbol{k} / 2}^{c}}{E_{\boldsymbol{p}+\boldsymbol{k} / 2}^{c}-E_{\boldsymbol{p}-\boldsymbol{k} / 2}^{c}-\hbar(\omega+i \eta)} .
\end{aligned}
$$

$f_{\boldsymbol{p}}^{c}$ is the momentum distribution function of species $c$. Again, a small but finite imaginary frequency $i \eta, \quad \eta>$ 0 has to be introduced, in order to fix the sign of the imaginary part and to obtain convergent results. In the case of a classical two-component plasma with different temperatures $T_{c}$, we take the Maxwell distribution. In the limit $\hbar \rightarrow 0$ the resulting susceptibility reads

$$
\chi_{c}^{0, \mathrm{cl}}(k, \omega)=-\Omega_{0} n_{c} W\left(x_{c}\right) / k_{\mathrm{B}} T_{c} .
$$

with the plasma dispersion function

$$
W\left(x_{c}\right)=1-2 x_{c} \mathrm{e}^{-x_{c}^{2}} \int_{0}^{x_{c}} \mathrm{e}^{t^{2}} \mathrm{~d} t-i \sqrt{\pi} x_{c} \mathrm{e}^{-x_{c}^{2}},
$$

and the dimensionless variable $x_{c}=\sqrt{\omega^{2} m_{c} / 2 k^{2} k_{\mathrm{B}} T_{c}}$. Eqs. (27) and (28) can be solved algebraically for the 
RPA-susceptibilities $\chi_{c c^{\prime}}^{\mathrm{RPA}}(\boldsymbol{k}, \omega)$ as shown in App. B see also Ref. [39]. Evaluation of Eq. (26) with $\chi_{\mathrm{ee}}^{\mathrm{RPA}}(\boldsymbol{k}, \omega)$, see Eqs. (29), and (B5), yields the electronic DSF in RPA, i.e.

$$
\begin{aligned}
& S_{\mathrm{ee}}(\boldsymbol{k}, \omega)= \\
& \left|\frac{1+Z_{\mathrm{eff}} \alpha^{2}\left(T_{\mathrm{e}} / T_{\mathrm{i}}\right) W\left(x_{\mathrm{i}}\right)}{1+\alpha^{2} W\left(x_{\mathrm{e}}\right)+\alpha^{2} Z_{\mathrm{eff}}\left(T_{\mathrm{e}} / T_{\mathrm{i}}\right) W\left(x_{\mathrm{i}}\right)}\right|^{2} \frac{x_{\mathrm{e}} \exp \left(-x_{\mathrm{e}}^{2}\right)}{\omega \sqrt{\pi}}+ \\
& +Z_{\mathrm{eff}}\left|\frac{-\alpha^{2} W\left(x_{\mathrm{e}}\right)}{1+\alpha^{2} W\left(x_{\mathrm{e}}\right)+\alpha^{2} Z_{\mathrm{eff}}\left(T_{\mathrm{e}} / T_{\mathrm{i}}\right) W\left(x_{\mathrm{i}}\right)}\right|^{2} \frac{x_{\mathrm{i}} \exp \left(-x_{\mathrm{i}}^{2}\right)}{\omega \sqrt{\pi}} .
\end{aligned}
$$

Here, the scattering parameter

$$
\alpha=\kappa_{\mathrm{e}} / k=\sqrt{n_{\mathrm{e}} e^{2} / m_{\mathrm{e}} k_{\mathrm{B}} T_{\mathrm{e}}} / k
$$

has been introduced. It separates the regime of collective Thomson scattering $(\alpha \gg 1)$, where collective resonances (plasmons) appear in the spectrum, and the regime of noncollective Thomson scattering $(\alpha \ll 1)$, where the spectrum reflects the single-particle distribution function of electrons without further structures. Expression (32) was also used by Baldis et al. in [16].

Eq. (32) for the DSF contains two terms: The first term is basically the DSF of a free electron gas. However, it also contains the ionic dispersion function, which accounts for the screening effect of ions. The second term gives the scattering signal from electrons which are close to ions and are therefore determined by the dynamics of these heavy particles. This term is dominant at small $\omega$ where $W\left(x_{\mathrm{e}}\right)$ can be approximated by its static limit, i.e. $W\left(x_{\mathrm{e}} \ll 1\right) \rightarrow 1$. For larger values of $\omega$, the first term dominates the spectrum, because the ion part is damped out more rapidly than the electron part. In particular, plasmon resonances, which appear in the spectrum due to a vanishing real part of the denominator, only survive in the first term, while they are damped out in the second. In so-called Salpeter approximation [40] the DSF (32) is separated into two terms depending only on electronic and on ionic variables respectively, electron-ion correlations are neglected to a large extent. Therefore, the full expression (32) should be used, as done throughout this work.

Chihara 22] gives the DSF in terms of the local field correction factor, thereby including electron-ion collisions. In appropriate limits, his expression coincides with Eq. (32), see Ref. [21]. On the other hand, due to the equivalence of the local field correction and the collision frequency in the high frequency limit, Chihara's expression leads to the same formula for the absorption coefficient $\alpha(\omega)$ as given in Eq. (8) [41]. Thus, Chihara presents an alternative approach to the question of photoabsorption and emission and Thomson scattering in plasmas starting from the DSF, whereas in this work the dielectric function is the central quantity.

Having the thermal emission spectrum (20) and the dynamic structure factor (32) at our disposal, we are now in the position to compare the signal from Thomson scattering to the bremsstrahlung background thereby treating both processes on a common basis.

\section{RESULTS}

We now compare power spectra for Thomson scattering and bremsstrahlung emission. Figures [3 - 6] show results for different combinations of electron density and temperature for a hydrogenic plasma. We consider densities $n_{\mathrm{e}}=10^{20} \mathrm{~cm}^{-3}$ and $10^{22} \mathrm{~cm}^{-3}$ and temperatures of $10 \mathrm{eV}$ and $50 \mathrm{eV}$ as examples for possible experimental conditions with cryogenic targets. Furthermore, backscattering geometry (scattering angle $120^{\circ}$ ) is chosen and a VUV laser wavelength of $\lambda=32 \mathrm{~nm}$ is assumed.

To model the detector, we assume a detector function $G(\lambda)$ of gaussian shape, its width given by the relative spectral bandwidth $\Delta \lambda / \lambda=\Delta \omega / \omega[16]$

$$
G(\lambda)=\frac{1}{\sqrt{2 \pi \sigma^{2}}} \mathrm{e}^{-\lambda^{2} / 2 \sigma^{2}}, \quad \sigma=0.425 \Delta \lambda .
$$

For the case of bremsstrahlung, we neglect the effect of the finite bandwidth of the detector, since the corresponding spectrum is only slowly varying with frequency. The effective ion charge $Z_{\text {eff }}$, calculated with COMPTRA04 [30] is close to $Z_{\text {eff }}=1$ in the case of hydrogen for the present values of electron density and temperature.

In Fig. 3. the black dashed curve represents the pure Thomson signal, i.e. no convolution with the detector function $G(\lambda)$ has been performed. For the present parameters $n_{\mathrm{e}}=10^{20} \mathrm{~cm}^{-3}$, and $k_{\mathrm{B}} T_{\mathrm{e}}=10 \mathrm{eV}$, the Thomson spectrum contains a very narrow ion peak, situated at the laser wavelength, and two satellites, which can be identified as electronic plasmon resonances. The central peak dominates these electronic resonances by a factor of 10, approximately. The solid black curve is obtained by convolution of the pure Thomson signal (dotted black curve) with the detector function. The detector resolution is set to $\Delta \lambda=3.2 \mathrm{~nm}$, which corresponds to $1 \%$ of the central wavelength. Due to the broad detector function, the central peak is lowered and broadened such that the plasmon resonances do not show up as separate structures anymore. Instead, they only provide sidewings in the spectrum. In Fig. 目 $\left(n_{\mathrm{e}}=10^{22} \mathrm{~cm}^{-3}\right.$, and $k_{\mathrm{B}} T_{\mathrm{e}}=10 \mathrm{eV}$ ), the electronic peaks are totally absorbed in the central peak. In this case, the ion peak in the unconvoluted signal (not shown) is several orders of magnitude larger than the electronic resonances, which therefore do not contribute to the convolution integral (3). Fig. 5 shows spectra for $n_{\mathrm{e}}=10^{20} \mathrm{~cm}^{-3}$, and $k_{\mathrm{B}} T_{\mathrm{e}}=50 \mathrm{eV}$. At these parameters, one gets $\alpha=0.56<1$ for the scattering parameter, we are in the noncollective regime. Already the unconvoluted Thomson signal is free of sharp electronic resonances, only two shoulders appear in the broad, noncollective signal. Finally, in Fig. [6 $\left(n_{\mathrm{e}}=10^{22} \mathrm{~cm}^{-3}\right.$, and $\left.k_{\mathrm{B}} T_{\mathrm{e}}=50 \mathrm{eV}\right)$, we 


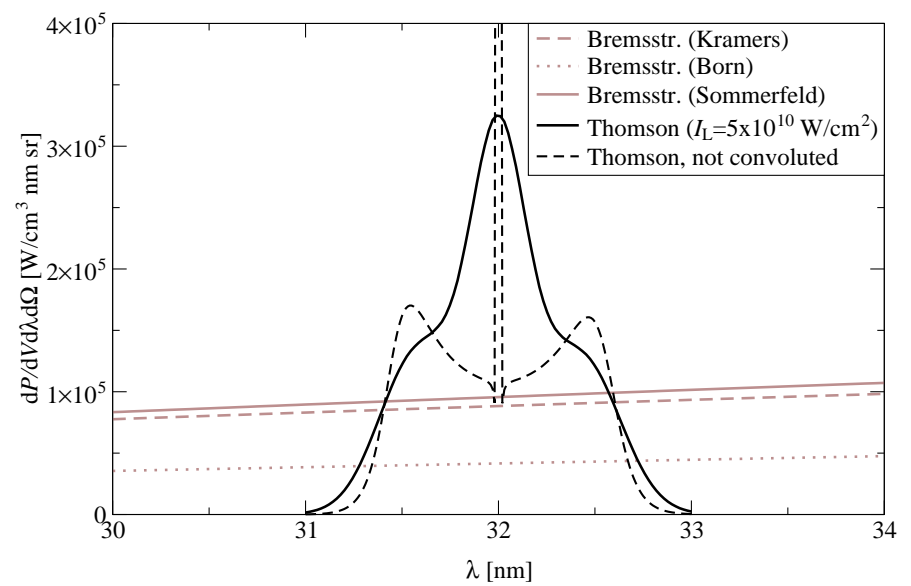

FIG. 3: Emission power spectra for Thomson scattering and bremsstrahlung. The black dashed curve represents the unconvoluted Thomson spectrum. Parameters: $k_{\mathrm{B}} T_{\mathrm{e}}=10 \mathrm{eV}$, $n_{\mathrm{e}}=10^{20} \mathrm{~cm}^{-3}$, laser wavelength $\lambda=32 \mathrm{~nm}$, scattering parameter $\alpha=1.25, Z_{\text {eff }}=1$.

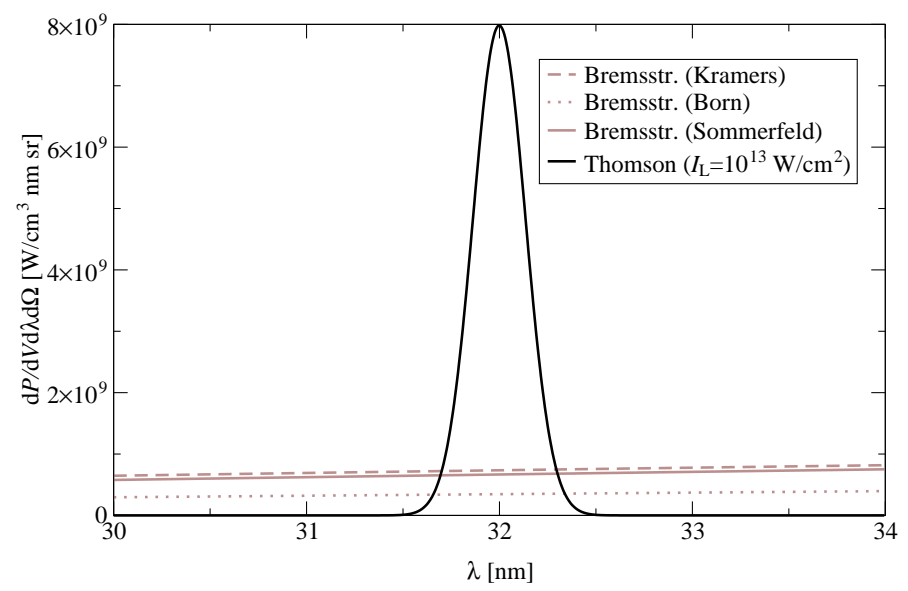

FIG. 4: Emission power spectra for Thomson scattering and bremsstrahlung. Parameters: $k_{\mathrm{B}} T_{\mathrm{e}}=10 \mathrm{eV}, n_{\mathrm{e}}=10^{22} \mathrm{~cm}^{-3}$, laser wavelength $\lambda=32 \mathrm{~nm}$, scattering parameter $\alpha=12.5$, $Z_{\text {eff }}=1$.

have the same structure as in Fig. 4 no plasmon peaks are visible due to the dominance of the ion feature.

In Figures [3 - 6] the intensity of the laser is chosen such that the central peak is situated clearly above the bremsstrahlung level. Three approximations for bremsstrahlung are shown, namely Kramers formula (19), Born approximation (18) and Sommerfeld's formula (22). Born approximation gives larger deviations from Kramers result than the Sommerfeld expression. This corresponds to the behaviour of the Gaunt factor shown in Fig. 2 The Sommerfeld result is always closer to unity than Born approximation.

In the following, we evaluate the threshold intensity $I_{\text {thresh }}(\omega)$ defined in Eq. (4) using Eqs. (3), (5), and (32)

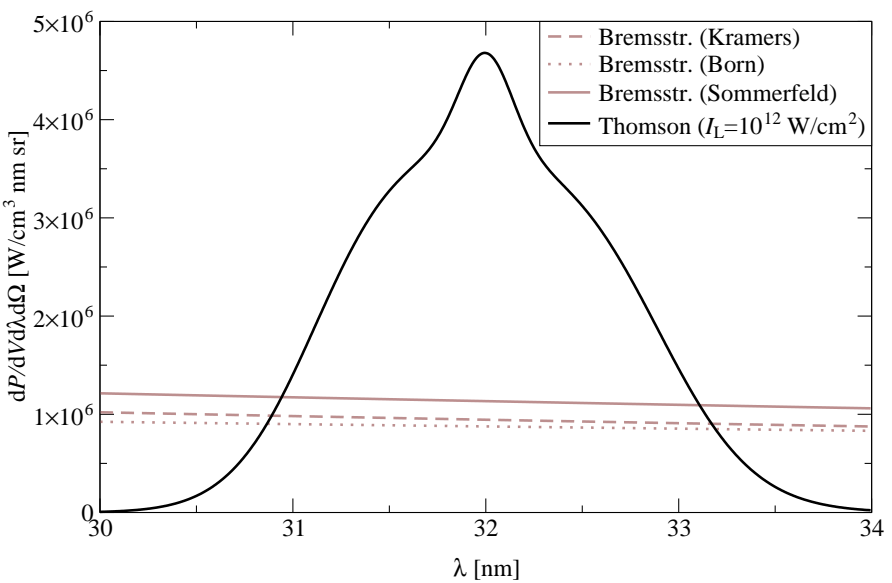

FIG. 5: Emission power spectra for Thomson scattering and bremsstrahlung. Parameters: $k_{\mathrm{B}} T_{\mathrm{e}}=50 \mathrm{eV}, n_{\mathrm{e}}=10^{20} \mathrm{~cm}^{-3}$, laser wavelength $\lambda=32 \mathrm{~nm}$, scattering parameter $\alpha=0.56$, $Z_{\text {eff }}=1$.

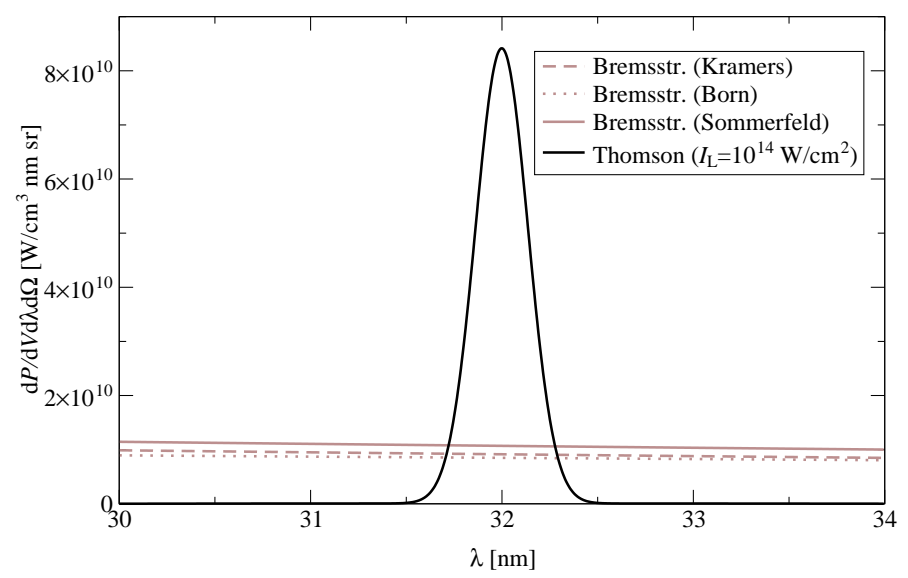

FIG. 6: Emission power spectra for Thomson scattering and bremsstrahlung. Parameters: $k_{\mathrm{B}} T_{\mathrm{e}}=50 \mathrm{eV}, n_{\mathrm{e}}=10^{22} \mathrm{~cm}^{-3}$, laser wavelength $\lambda=32 \mathrm{~nm}$, scattering parameter $\alpha=5.6$, $Z_{\text {eff }}=1$.

for the Thomson power spectrum and Eq. (20) with the Gaunt factor in either Born approximation (21) or tmatrix approximation (22) for the bremsstrahlung power spectrum.

Figures [7- 10] show contour plots of the threshold intensity in part of the $n_{\mathrm{e}}-T_{\mathrm{e}}$-plane. In Fig. 7 we compare Kramers result (dashed curves) to Born approximation (dotted curves) assuming aluminum as target material, scattering angle $\theta=20^{\circ}, \Delta \lambda / \lambda=10^{-4}$. In this case, the effective ion charge $Z_{\text {eff }}$ is calculated using Thomas-Fermi theory [29] as was also done by Baldis et al. in Ref. 16], who used the same set of parameters. Their results are reproduced by using Kramers formula for bremsstrahlung (dashed curve).

For low temperatures, where the Gaunt factor in Born 
approximation is smaller than unity at the considered photon energy, cf. Fig. 2, higher densities are accessible as compared to Kramers result. For high temperatures, the opposite becomes true, $\bar{g}^{\mathrm{B}}(\omega)>1$ leads to lower accessible densities.

In figures [ - 10] three approximations have been calculated for the bremsstrahlung level. Besides Kramers formula (dashed curves) we show results for Born approximation (dotted curves) and Sommerfeld's formula (solid curves). Comparing the Sommerfeld result to Born approximation, it can be noted that Born approximation gives larger corrections, while Sommerfeld's theory leads to smaller deviations from Kramers result. This is one important result of this work: Taking into account quantum effects in a rigorous way via Sommerfeld's expression for the Gaunt factor leads only to small corrections of threshold intensities, while Born approximation tends to overestimate these effects. This underlines the importance to go beyond Born approximation. For moderate and high temperatures, the Sommerfeld result lies systematically below the Kramers result, due to the increasing Gaunt factor at high temperatures.

Furthermore, we investigated the influence of other experimental parameters, namely the laser wavelength, the material, and the spectral bandwidth of the spectrometer. The latter parameter turns out to be of great importance: By comparison of Fig. 8] with Fig. 9] one observes that notably higher densities are accessible in the case of small spectral bandwidth $\left(\Delta \lambda / \lambda=10^{-4}\right.$ in Fig. (9) than for the relatively large bandwidth $\left(\Delta \lambda / \lambda=10^{-2}\right.$ in Fig. [8).

Comparing Fig. 7 to Fig. 9] the influence of the $Z$-number of the material becomes apparent: Low $Z$-materials produce less bremsstrahlung than high $Z$-elements due to the $Z^{2}$-proportionality of the bremsstrahlung cross section, cf. Eq. (19). On the other hand, the $Z$-dependance of the dynamical structure factor largely cancels out for the free electron part of the structure factor while it is roughly $Z /(1+Z)^{2}$ for the ionic part, cf. Eq. (32).

Finally, important differences are noted upon changing the laser wavelength from $32 \mathrm{~nm}$ (Fig. 9) to $13 \mathrm{~nm}$ (Fig. 10) especially at low temperatures. Both data sets have been calculated using the same spectral resolution $\left(\Delta \lambda / \lambda=10^{-4}\right)$ and material $(\mathrm{H})$. Looking at Fig. 10] $(\lambda=13 \mathrm{~nm})$ at low temperatures the threshold contours are almost parallel to the density axis. For higher temperatures, both wavelengths $32 \mathrm{~nm}$ and $13 \mathrm{~nm}$ give nearly equal threshold intensities, which do not depend on temperature. This can be understood from the bremsstrahlung spectrum, which becomes nearly independent of frequency and temperature at low $\hbar \omega / k_{\mathrm{B}} T$.

Since the information about temperature and density is stored in the position and height of the plasmon resonances, we will now focus on experimental conditions to be met in order to separate the plasmon peak from bremsstrahlung background. Results for the threshold intensity are given in Tab. I] The electron density is set

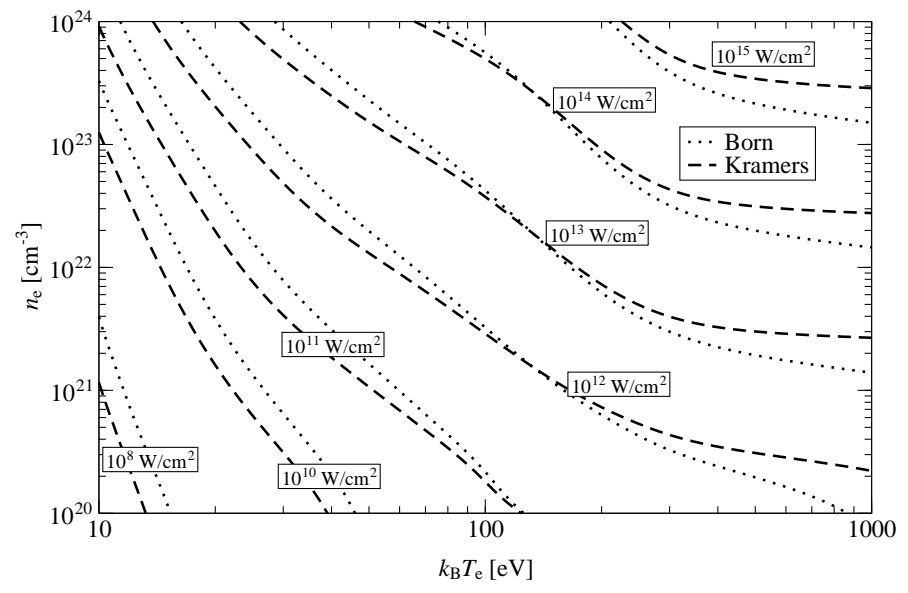

FIG. 7: Threshold curves in the density-temperature plane for Al. Parameters: laser wavelength $14.7 \mathrm{~nm}, \theta=20^{\circ}, \Delta \lambda / \lambda=$ $10^{-4}, T_{\mathrm{e}} / T_{\mathrm{i}}=2$.

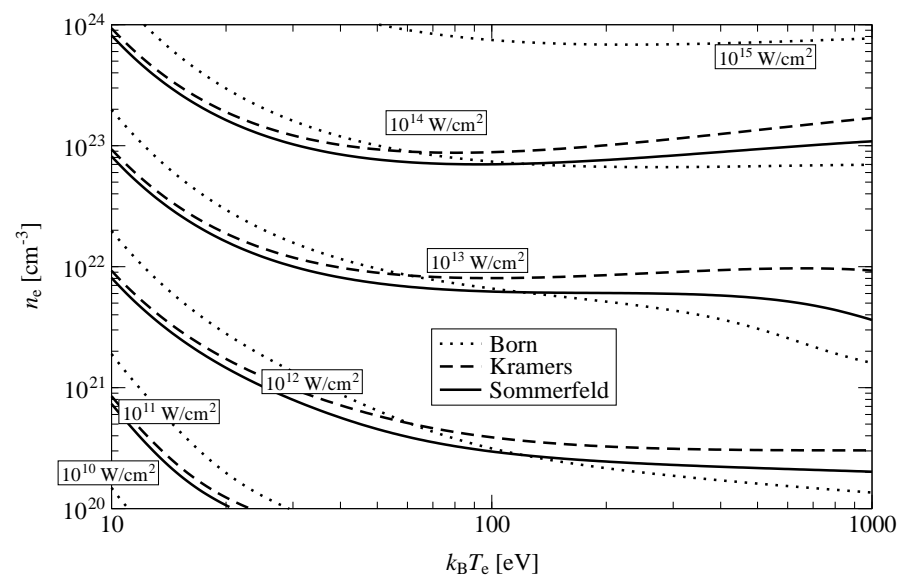

FIG. 8: Threshold curves in the density-temperature plane for H. Parameters: laser wavelength $32 \mathrm{~nm}, \theta=120^{\circ}, \Delta \lambda / \lambda=$ $10^{-2}, T_{\mathrm{e}} / T_{\mathrm{i}}=2$.

to $n_{\mathrm{e}}=10^{21} \mathrm{~cm}^{-3}$, for $n_{\mathrm{e}}=10^{20} \mathrm{~cm}^{-3}$ and $10^{22} \mathrm{~cm}^{-3}$ see figures 3-6 Two wavelengths are considered, $\lambda=32 \mathrm{~nm}$, the momentary VUV-FEL wavelength at DESY, and $\lambda=13 \mathrm{~nm}$, envisaged wavelength in the near future. Since the latter wavelength allows for very efficient $\mathrm{x}$ ray optics to be applied, the spectral resolution has been reduced to $\Delta \lambda / \lambda=10^{-4}$, while $32 \mathrm{~nm}$ wavelength allows only for $\Delta \lambda / \lambda=10^{-2}$. Finally the scattering angle has been chosen such that the plasmon peak is on the one hand well pronounced but on the other hand shifted far enough from the central peak as to be resolved by the spectrometer. For detailed discussion of the Thomson scattering spectrum at various plasma parameters, we refer to Refs. [12, 13]. The rightmost column of Tab. I gives the Gaunt factor in Sommerfeld approximation (Eq. (22)). Note that in the case of the $32 \mathrm{~nm}$ wavelength, the threshold intensity is increased by $14-22 \%$ 


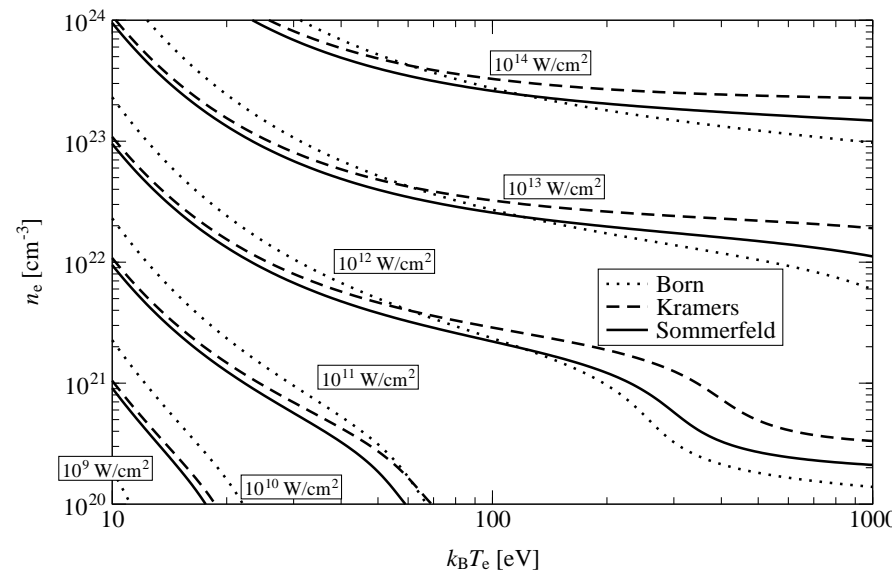

FIG. 9: Threshold curves in the density-temperature plane for H. Parameters: laser wavelength $32 \mathrm{~nm}, \theta=120^{\circ}, \Delta \lambda / \lambda=$ $10^{-4}, T_{\mathrm{e}} / T_{\mathrm{i}}=2$.

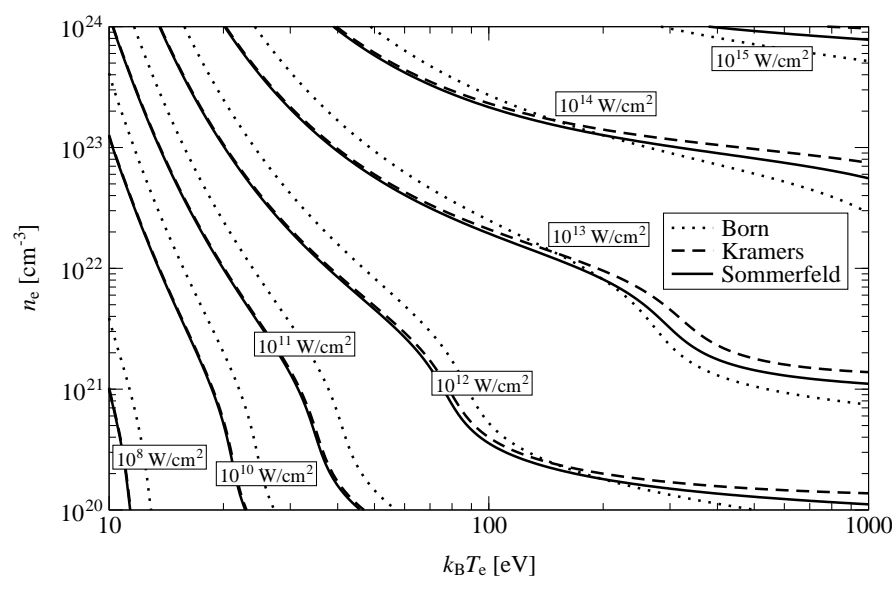

FIG. 10: Threshold curves in the density-temperature plane for $\mathrm{H}$. Parameters: laser wavelength $13.0 \mathrm{~nm}, \theta=120^{\circ}$, $\Delta \lambda / \lambda=10^{-4}, T_{\mathrm{e}} / T_{\mathrm{i}}=2$.

due to the Gaunt factor.

\section{CONCLUSION}

In this work we have studied Thomson scattering and bremsstrahlung emission in warm dense matter in the context of plasma diagnostic experiments to be performed in near future. Out of these two competing, Thomson scattering is supposed to serve as a probe for plasma parameters, but also bremsstrahlung gives an important contribution to the photon yield from highly ionized plasmas. Thus, experimental conditions have to be determined that allow for a maximum signal-tobackground ratio. Here, we focused on the laser intensity. To this purpose, expressions for the Thomson signal, which is given by the dynamical structure factor of
TABLE I: Experimental parameters and resulting threshold intensities for different electron temperatures and laser wavelengths. Fixed parameters: $Z=1, n_{\mathrm{e}}=10^{21} \mathrm{~cm}^{-3}$.

\begin{tabular}{|c|c|c|c|c|c|c|}
\hline$k_{\mathrm{B}} T_{\mathrm{e}}[\mathrm{eV}]$ & $\lambda[\mathrm{nm}]$ & $\theta$ & $\Delta \lambda / \lambda$ & $\lambda_{\mathrm{pl}}[\mathrm{nm}]$ & $I_{\mathrm{thresh}}\left[\mathrm{W} / \mathrm{cm}^{2}\right]$ & $\bar{g}_{\mathrm{ff}}^{\mathrm{S}}$ \\
\hline 10 & 32 & 120 & $10^{-2}$ & 33.1 & $1.37 \cdot 10^{12}$ & 1.14 \\
20 & 32 & 120 & $10^{-2}$ & 33.2 & $4.92 \cdot 10^{12}$ & 1.17 \\
50 & 32 & 120 & $10^{-2}$ & 33.4 & $9.29 \cdot 10^{12}$ & 1.22 \\
\hline 10 & 13 & 120 & $10^{-4}$ & 13.3 & $2.95 \cdot 10^{9}$ & 1.03 \\
20 & 13 & 45 & $10^{-4}$ & 13.2 & $1.55 \cdot 10^{11}$ & 1.04 \\
50 & 13 & 45 & $10^{-4}$ & 13.2 & $3.49 \cdot 10^{12}$ & 1.06 \\
\hline
\end{tabular}

the plasma as well as for the bremsstrahlung spectrum have been derived from a common starting point, namely linear response theory. This approach allows for a systematic treatment of medium and quantum effects, such as dynamical screening and strong collisions.

We have applied our formulas to determine threshold intensities for the external photon source (FEL) as function of density and temperature as well as their dependence on further parameters, namely the laser wavelength, the spectral resolution of detectors, and the material. In the discussion we focused on the bremsstrahlung spectrum. It was shown that Born approximation overestimates the effect of collisions, i.e. it leads to relatively high Gaunt factors if compared to Sommerfeld's expression. Sommerfeld's formula (22) gives the correct Gaunt factor in a weakly coupled plasma. At temperatures $k_{\mathrm{B}} T_{\mathrm{e}} \geq 20 \mathrm{eV}$ threshold intensities calculated with the Gaunt factor in Sommerfeld approximation are larger than those obtained by Baldis et al. in Ref. [16], who used Kramers expression, i.e. $\bar{g}_{\mathrm{ff}}=1$.

The Thomson signal was analysed within RPA which gives the contribution in lowest order of density. For densities investigated here, collisions are also relevant for the Thomson signal and lead to a pronounced change of the electronic part in $S(\boldsymbol{k}, \lambda)$. This was investigated in Ref. [42]. However, the account for collisions does not alter the results for the threshold intensities performed in this work, since the Thomson scattering signal was evaluated at the laser wavelength, were the ionic feature of the DSF dominates.

Finally, we have demonstrated, that Thomson scattering can easily overcome the bremsstrahlung background, if laser intensities of $10^{8} \mathrm{~W} / \mathrm{cm}^{2}-10^{13} \mathrm{~W} / \mathrm{cm}^{2}$ are provided. From this point of view, Thomson scattering experiments for plasma diagnostics using VUV-FEL radiation seem feasible.

\section{Acknowledgments}

This work has been supported by the Virtual Institute VH-VI-104 Plasma Physics Research using FEL Radiation of the Helmholtz Society and the DFGSonderforschungsbereich 652 Starke Korrelationen und kollektive Phänomene im Strahlungsfeld: Coulombsys- 
teme, Cluster und Partikel. We gratefully acknowledge A. Höll and J. Chihara for stimulating discussion. C.F. would like to thank DESY for hospitality and support during a two week visit.

\section{APPENDIX A: CORRELATION FUNCTIONS AND GREEN FUNCTIONS}

Correlation functions for two observables $A, B$ are defined according to

$$
\begin{aligned}
(A ; B) & =\frac{1}{\beta} \int_{0}^{\beta} \mathrm{d} \tau \operatorname{Tr}\left[A(-i \hbar \tau) B^{\dagger} \rho_{0}\right], \\
\langle A ; B\rangle_{\omega+i \eta} & =\int_{0}^{\infty} \mathrm{d} t \mathrm{e}^{i(\omega+i \eta) t}(A(t) ; B),
\end{aligned}
$$

$\rho_{0}$ is the equilibrium statistical operator. The time dependence of observables is taken in the Heisenberg picture, using the system Hamiltionian

$$
H=\sum_{\boldsymbol{p}, c} \frac{\hbar^{2} p^{2}}{2 m_{c}} a_{\boldsymbol{p}, c}^{\dagger} a_{\boldsymbol{p}, c}+\frac{1}{2} \sum_{\boldsymbol{p} \boldsymbol{k \boldsymbol { q } , c d}} V_{c d}(\boldsymbol{q}) a_{\boldsymbol{p}+\boldsymbol{q}, c}^{\dagger} a_{\boldsymbol{k}-\boldsymbol{q}, d}^{\dagger} a_{\boldsymbol{k}, d} a_{\boldsymbol{p}, c} .
$$

Performing integration by parts, the current-current correlation function (16) can be expressed through a forceforce correlation function as

$$
\chi^{-1}(\boldsymbol{k}, \omega)=\frac{i}{\beta \Omega_{0}} \frac{\omega q^{2}}{k^{2}} \frac{1}{\left(J_{k}^{z} ; J_{k}^{z}\right)^{2}}\left[-i \omega\left(J_{k}^{z} ; J_{k}^{z}\right)+\left\langle\dot{j}_{k}^{z} ; \dot{j}_{k}^{z}\right\rangle_{\omega+i \eta}-\frac{\left\langle\dot{J}_{k}^{z} ; J_{k}^{z}\right\rangle_{\omega+i \eta}\left\langle J_{k}^{z} ; \dot{J}_{k}^{z}\right\rangle_{\omega+i \eta}}{\left\langle J_{k}^{z} ; J_{k}^{z}\right\rangle_{\omega+i \eta}}\right],
$$

with $\dot{J}_{0, \mathrm{e}}^{z}=i\left[H, J_{0, \mathrm{e}}^{z}\right] / \hbar$.

Also, it is convenient to introduce a generalized collision frequency $\nu(\omega)$ in analogy to the Drude relation [20] $\epsilon(\omega)=1-\omega_{\mathrm{pl}}^{2} /\left(\omega^{2}+i \omega \nu(\omega)\right)$ where $\omega_{\mathrm{pl}}^{2}=$ $\sum_{c} n_{c} e_{c}^{2} /\left(\epsilon_{0} m_{c}\right)$ is the squared plasma frequency.

By comparison with Eq. (12) using $\left(J_{k}^{z} ; J_{k}^{z}\right)=\epsilon_{0} \omega_{\mathrm{pl}}^{2} / \beta \Omega_{0}$, we establish an expression for the collision frequency in terms of correlation functions

$$
\begin{aligned}
\nu(\omega)=\frac{\beta \Omega_{0}}{\epsilon_{0} \omega_{\mathrm{pl}}^{2}} \lim _{k \rightarrow 0} & {\left[\left\langle\dot{J}_{k}^{z}, \dot{J}_{k}^{z}\right\rangle_{\omega+i \eta}\right.} \\
& \left.-\frac{\left\langle\dot{J}_{k}^{z} ; J_{k}^{z}\right\rangle_{\omega+i \eta}\left\langle J_{k}^{z} ; \dot{J}_{k}^{z}\right\rangle_{\omega+i \eta}}{\left\langle J_{k}^{z} ; J_{k}^{z}\right\rangle_{\omega+i \eta}}\right] .
\end{aligned}
$$

Further details can be found in Ref. 20]. Making use of Eq. (8), the absorption coefficient can be expressed as

$$
\alpha(\omega)=\frac{\omega_{\mathrm{pl}}^{2}}{c} \frac{\operatorname{Re} \nu(\omega)}{\left(\omega^{2}-2 \omega \operatorname{Im} \nu(\omega)+|\nu(\omega)|^{2}\right) n(\omega)} .
$$

In the high frequency limit $\omega \gg \omega_{\mathrm{pl}}$, the index of refraction is unity, the imaginary part of the collision frequency tends to zero and the collision frequency is small compared to the frequency $\omega$. Then, we can consider the approximation $\alpha(\omega)=\omega_{\mathrm{pl}}^{2} \operatorname{Re} \nu(\omega) / \omega^{2} c=$ $\beta \Omega_{0} \operatorname{Re}\left\langle\dot{J}_{0}^{z}, \dot{J}_{0}^{z}\right\rangle_{\omega+i \eta} / c \epsilon_{0} \omega^{2}$, where the collision frequency is given in the form of a force-force correlation function, cf. Ref. [20]. Thus, the absorption coefficient is directly proportional to the real part of the force-force correlation function, which itself can be determined using perturbation theory.

The relation to the thermodynamic Green function of two observables is given by

$$
\langle A ; B\rangle_{\omega+i \eta}=\frac{i}{\pi \beta} \int_{-\infty}^{\infty} \frac{\mathrm{d} \bar{\omega}}{\bar{\omega}} \frac{1}{\omega+i \eta-\bar{\omega}} \operatorname{Im} G_{A B}(\bar{\omega}+i \eta) .
$$

Using Dirac's identity one obtains $\operatorname{Re}\langle A ; B\rangle_{\omega+i \eta}=$ $\operatorname{Im} G_{A B}(\omega+i \eta) / \omega \beta$, which directly leads to Eq. (16).

\section{APPENDIX B: THE RPA-SUSCEPTIBILITY}

Eq. (27) is obtained from the exact relation for the response function $\chi_{c c^{\prime}}(\boldsymbol{k}, \omega)$,

$\chi_{c c^{\prime}}(\boldsymbol{k}, \omega)=\chi_{c}^{0}(\boldsymbol{k}, \omega) \delta_{c c^{\prime}}+\sum_{d} \chi_{c}^{0}(\boldsymbol{k}, \omega) V_{c d}^{\mathrm{sc}}(\boldsymbol{k}, \omega) \chi_{d c^{\prime}}(\boldsymbol{k}, \omega)$,

by truncation after the first iteration, i.e. $\chi_{d c^{\prime}}=\chi_{d c^{\prime}}^{0}$ in the second term of Eq. (B1). Insertion of the screened potential Eq. (28) yields the closed equation 


$$
\begin{aligned}
\chi_{c c^{\prime}}^{\mathrm{RPA}}(\boldsymbol{k}, \omega) & =\chi_{c}^{0}(\boldsymbol{k}, \omega) \delta_{c c^{\prime}}+\chi_{c}^{0}(\boldsymbol{k}, \omega)\left[V_{c c^{\prime}}(\boldsymbol{k})+\sum_{d} V_{c d}(\boldsymbol{k}) \chi_{d}^{0}(\boldsymbol{k}, \omega) V_{d c^{\prime}}^{\mathrm{sc}}(\boldsymbol{k}, \omega)\right] \chi_{c^{\prime}}^{0}(\boldsymbol{k}, \omega) \\
& =\chi_{c}^{0}(\boldsymbol{k}, \omega) \delta_{c c^{\prime}}+\sum_{d} \chi_{c}^{0}(\boldsymbol{k}, \omega) V_{c d}(\boldsymbol{k}) \chi_{d c^{\prime}}^{\mathrm{RPA}}(\boldsymbol{k}, \omega) .
\end{aligned}
$$

For a two component plasma, i.e. an electron-ion plasma $c=\mathrm{e}$, i, we obtain by matrix inversion

$$
\begin{aligned}
\chi_{\mathrm{ee}}^{\mathrm{RPA}} & =\frac{\chi_{\mathrm{e}}^{0}\left(1-\chi_{\mathrm{i}}^{0} V_{\mathrm{ii}}\right)}{1-\chi_{\mathrm{e}}^{0} V_{\mathrm{ee}}-\chi_{\mathrm{i}}^{0} V_{\mathrm{ii}}+\chi_{\mathrm{i}}^{0} \chi_{\mathrm{e}}^{0}\left(V_{\mathrm{ii}} V_{\mathrm{ee}}-V_{\mathrm{ei}} V_{\mathrm{ie}}\right)}, \\
\chi_{\mathrm{ie}}^{\mathrm{RPA}} & =\frac{\chi_{\mathrm{e}}^{0} V_{\mathrm{ei}} \chi_{\mathrm{i}}^{0}}{1-\chi_{\mathrm{e}}^{0} V_{\mathrm{ee}}-\chi_{\mathrm{i}}^{0} V_{\mathrm{ii}}+\chi_{\mathrm{i}}^{0} \chi_{\mathrm{e}}^{0}\left(V_{\mathrm{ii}} V_{\mathrm{ee}}-V_{\mathrm{ei}} V_{\mathrm{ie}}\right)} .
\end{aligned}
$$

$\chi_{\mathrm{ei}}^{\mathrm{RPA}}$ and $\chi_{\mathrm{ii}}^{\mathrm{RPA}}$ are obtained by interchanging indices $\mathrm{i}$ and e in Eq. (B4) and Eq. (B3), respectively.

The imaginary part of the electronic susceptibility in RPA, $\chi_{\mathrm{ee}}^{\mathrm{RPA}}(\boldsymbol{k}, \omega)$ (Eq. (B3) ) for a hydrogen plasma $\left(V_{\mathrm{ee}}=V_{\mathrm{ii}}=-V_{\mathrm{ei}}=-V_{\mathrm{ie}} \equiv V\right)$ now evaluates to

$$
\begin{aligned}
& \operatorname{Im} \chi_{\mathrm{ee}}^{\mathrm{RPA}}(\boldsymbol{k}, \omega)= \\
& \quad\left|\frac{1-V(\boldsymbol{k}) \chi_{\mathrm{i}}^{0}(\boldsymbol{k}, \omega)}{1-V(\boldsymbol{k})\left[\chi_{\mathrm{e}}^{0}(\boldsymbol{k}, \omega)+\chi_{\mathrm{i}}^{0}(\boldsymbol{k}, \omega)\right]}\right|^{2} \operatorname{Im} \chi_{\mathrm{e}}^{0}(\boldsymbol{k}, \omega)+ \\
& \quad+\left|\frac{V(\boldsymbol{k}) \chi_{\mathrm{e}}^{0}(\boldsymbol{k}, \omega)}{1-V(\boldsymbol{k})\left[\chi_{\mathrm{e}}^{0}(\boldsymbol{k}, \omega)+\chi_{\mathrm{i}}^{0}(\boldsymbol{k}, \omega)\right]}\right|^{2} \operatorname{Im} \chi_{\mathrm{i}}^{0}(\boldsymbol{k}, \omega) .
\end{aligned}
$$

This expression can also be derived from kinetic theory, i.e. the perturbative expansion of Vlasov's equation for a two component plasma [15]. It was used in [16] to compare the Thomson signal to the emission background caused by thermal bremsstrahlung photons. Generalizing for different electron and ion temperatures $T_{\mathrm{e}}$ and $T_{\mathrm{i}}$ and inserting the explicit expression for the response function in RPA (30), one obtains Eq. (32).
[1] S. C. Snyder, L. D. Reynolds, G. D. Lassahn, J. R. Fincke, C. B. Shaw, Jr, and R. J. Kearney, Phys. Rev. E 47, 1996 (1993).

[2] S. C. Snyder, G. D. Lassahn, and L. D. Reynolds, Phys. Rev. E 48, 4124 (1993).

[3] S. C. Snyder, L. D. Reynolds, J. R. Fincke, G. D. Lassahn, J. D. Grandy, and T. E. Repetti, Phys. Rev. E 50, 519 (1994).

[4] R. E. Bentley, J. Phys. D 30, 2880 (1997).

[5] C. Chenais-Popovics, V. Malka, J.-C. Gautier, S. Gary, O. Peyrusse, M. Rabec-Le Gloahec, I. Matsushima, C. Bauche-Arnoult, A. Bachelier, and J. Bauche, Phys. Rev. E 65, 046418 (2002).

[6] A. B. Murphy, Phys. Rev. E 69, 016408 (2004).

[7] D. E. Evans, Plasma Phys. 12, 573 (1970).

[8] S. H. Glenzer, G. Gregori, R. W. Lee, F. J. Rogers, S. W. Pollaine, and O. L. Landen, Phys. Rev. Lett. 90, 175002 (2003).

[9] G. Gregori, S. H. Glenzer, W. Rozmus, R. W. Lee, and O. L. Landen, Phys. Rev. E 67, 026412 (2003).

[10] G. Gregori, S. H. Glenzer, F. J. Rogers, S. M. Pollaine,
O. L. Landen, C. Blancard, G. Faussurier, P. Renaudin, S. Kuhlbrodt, and R. Redmer, Phys. Plasmas 11, 2754 (2004).

[11] V. Ayvazyan et al., Eur. Phys. J. D 37, 297 (2006).

[12] R. Redmer, H. Reinholz, G. Röpke, R. Thiele, and A. Höll, IEEE Trans. Plasma. Sci. 33, 77 (2005).

[13] A. Höll, R. Redmer, G. Röpke, and H. Reinholz, Eur. Phys. J. D 29, 159 (2004).

[14] H. A. Kramers, Philos. Mag. 46, 836 (1923).

[15] D. E. Evans and J. Katzenstein, Rep. Prog. Phys. 32, 207 (1969).

[16] H. A. Baldis, J. Dunn, M. E. Foord, and W. Rozmus, Rev. Sci. Instrum. 73, 4223 (2002).

[17] J. A. Gaunt, Proc. R. Soc. A 126, 654 (1930).

[18] D. Zubarev, V. Morozov, and G. Röpke, Statistical Mechanics of Nonequilibrium Processes, vol. 2 (Akademie Verlag, Berlin, 1996).

[19] A. Wierling, T. Millat, G. Röpke, R. Redmer, and H. Reinholz, Phys. Plasmas 8, 3810 (2001).

[20] H. Reinholz, R. Redmer, G. Röpke, and A. Wierling, Phys. Rev. E 62, 5648 (2000). 
[21] J. Chihara, J. Phys. F 17, 295 (1987).

[22] J. Chihara, J. Phys.: Condens. Matter 12, 231 (2000).

[23] C. Itzykson and J.-B. Zuber, Quantum field theory (McGraw-Hill, New York, 1980).

[24] S. Ichimaru, Statistical Plasma Physics, vol. 1 (AddisonWesley, New York, 1991).

[25] H. R. Griem, Principles of Plasma Spectroscopy (Cambridge University Press, Cambridge, 1997).

[26] G. D. Mahan, Many-Particle Physics (Plenum Press, New York and London, 1981), 2nd ed.

[27] A. Sommerfeld, Atombau und Spektrallinien, vol. 1 (Vieweg, Braunschweig, 1949).

[28] C. Fortmann, H. Reinholz, G. Röpke, and A. Wierling, in Condensed Matter Theory (2005), vol. 28, submitted, physics/0502051.

[29] R. M. More, Tech. Rep. UCRL-84991-1, Lawrence Livermore National Laboratory (1991).

[30] S. Kuhlbrodt, B. Holst, and R. Redmer, Contrib. Plasma Phys. 45, 73 (2005).

[31] J. D. Jackson, Classical Electrodynamics (J. Wiley \& Sons, New York, 1975), 2nd ed.

[32] W. Heitler, The Quantum Theory of Radiation (Dover
Publications, New York, 1984).

[33] M. Ter-Mikaelyan, Dokl. Akad. Nauk SSSR 94, 1033 (1953).

[34] M. Abramowitz and A. Stegun, eds., Handbook of Mathematical Functions with Formulas, Graphs and Mathematical Tables (Dover Publications, New York, 1970), 9th ed.

[35] W. J. Karzas and R. Latter, Astrophys. J. Suppl. Ser. 6, 167 (1961).

[36] C. W. Allen, Astrophysical Quantities (The Athlone Press, London, 1973).

[37] H. A. Gould and H. E. DeWitt, Phys. Rev. 155, 68 (1967).

[38] R. Kubo, J. Phys. Soc. Jpn. 12, 570 (1950).

[39] A. Selchow, G. Röpke, A. Wierling, H. Reinholz, T. Pschiwul, and G. Zwicknagel, Phys. Rev. E 64, 056410 (2001).

[40] E. E. Salpeter, Phys. Rev. 120, 1528 (1960).

[41] J. Chihara, private communication.

[42] R. Thiele, H. Reinholz, R. Redmer, and G. Röpke, J. Phys. A (accepted) (2006). 